\title{
ACCOUNT OF DYNAMIC MODELS OF ROTATORY MOTION OF SHIP AT PROVIDING OF SAFETY OF NAVIGATOR
}

\section{УЧЕТ ДИНАМИЧЕСКИХ МОДЕЛЕЙ ВРАЩАТЕЛЬНОГО ДВИЖЕНИЯ СУДНА ПРИ ОБЕСПЕЧЕНИИ БЕЗОПАСНОСТИ СУДОВОЖДЕНИЯ}

\author{
Y.V. Kazak, PhD student, G.E. Kalinichenko, PhD student \\ Ю.В. Казак, аспирант, Г. Е. Калиниченко, аспирант \\ National University «Odessa Maritime Academy», Ukraine \\ Национальный университет «Одесская морская академия», Украина
}

\begin{abstract}
It is shown that at sailing of ship in the compressed terms is achieved minimization of risks of origin of emergency situation at the use of dynamic model of rotatory motion of ship of the third order. Taking into account the use of this model procedure of determination of vector's position error and forming of region of dangerous ships is considered at the external process control of divergence.

It is marked in work, that at the turn of ship one of reasons of appearance of vector's error in relation to the next point of output of ship on a new course is the error of position angle of feather of helm, thus in the case of appearance of this error the resulted mechanism of forming of vector's error.

It is shown in the article, that rapprochement of two ships can be analyzed by the region of dangerous courses, that is represented on the plane of courses of ships, thus overhead and lower by the scopes of region there are points which meet condition of equality distance of the shortest rapprochement and maximum - possible distance. Points between the scopes of region answer the courses of ships, at which distance of the shortest rapprochement of more small after maximum - possible distance, that characterizes dangerous rapprochement of ships.

In the case of determination of scopes of region of dangerous courses without taking into account inertia of ships at a turn ships will divide in the distance, that more small after maximum - possible distance. Therefore, for the account of inertia of turn it is necessary maximum - to advance possible distance by the proper amendment. The size of this amendment is simply determined by the dynamic model of rotatory motion of ship, and accordance of value of amendment with true is determined by the degree of adequacy of dynamic model of rotatory motion of ship to the real process of turn of ship. Thus, the use of model of turning of ship depends on the degree of accordance of dynamic model of rotatory motion of ship to the real process of his turn.
\end{abstract}

The most adequate dynamic model of rotatory motion of ship, which is described by differential equalizations of the third order, is considered in work. 
It is marked that the turn of ship consists of two phases of laying of feather of helm. At first, on the first phase, position angle of helm which is contained in such position some time domain is conducted in the initial moment of time. Then position angle of helm is conducted in other side on that size of и inertia of turn of ship is extinguished. As a result, a ship goes out on the inflicted course, angular speed of turn diminishes to the zero, and the feather of helm is driven to the diametral plane of ship.

Keywords: safety of navigation, dynamic model of agility of ship, vector's error, region of dangerous courses.

\section{РЕФЕРАТ}

Показано, що при плаванні судна в стислих умовах мінімізація ризиків виникнення аварійної ситуації досягається при використанні динамічної моделі обертального руху судна третього порядку. 3 урахуванням використання цієї моделі розглянуто процедуру визначення векторіальної позиційної похибки і формування області небезпечних суден при зовнішньому управлінні процесом розходження.

В роботі відмічено, що при повороті судна однією із причин появи векторіальної похибки відносно прогнозуємої точки виходу судна на новий курс є похибка перекладки пера керма, причому в разі появи цієї похибки приведений механізм формування векторіальної похибки.

В статті показано, що зближення двох суден може аналізуватися областю небезпечних курсів, що відображається на площині курсів суден, при цьому верхньою і нижньою межами області є точки, які задовольняють умові рівності дистанції найкоротшого зближення та гранично - допустимої дистанції. Точки між межами області відповідають курсам суден, при яких дистанція найкоротшого зближення менша за гранично - допустиму дистанцію, що характеризує небезпечне зближення суден.

В разі визначення меж області небезпечних курсів без урахування інерційності суден при повороті судна розійдуться на відстані, що менша за гранично - допустиму дистанцію. Тому для урахування інерційності повороту необхідно гранично - допустиму дистанцію збільшити на відповідну поправку. Величина цієї поправки однозначно визначається динамічною моделлю обертального руху судна, а відповідність значення поправки до істинного визначається ступенем адекватності динамічної моделі обертального руху судна реальному процесу повороту судна. Таким чином, використання моделі поворотності судна залежить від ступеня відповідності динамічної моделі обертального руху судна реальному процесу його повороту.

В роботі розглянуто найбільш адекватну динамічну модель обертального руху судна, яка описується диференціальними рівняннями третього порядку.

Відзначено, що поворот судна складається із двох фаз кладки пера керма. Спочатку, на першій фазі, в початковий момент часу проводиться перекладка керма, яке утримується в такому положенні деякий інтервал часу. Потім проводиться перекладка керма в іншу сторону на ту ж величину и гаситься 
інерція повороту судна. В результаті судно виходить на завданий курс, кутова швидкість повороту зменшується до нуля, а перо керма приводиться в діаметральну площину судна.

Ключові слова: безпечність судноводіння, динамічна модель обертального руху судна, векторіальна похибка, область небезпечних курсів.

\section{Постановка проблемы в общем виде и ее связь с важными научными или практическими задачами}

Обеспечение безопасности судовождения в стесненных районах плавания является одной из наиболее актуальных проблем, решение которой способствует снижению числа навигационных аварий, возникающих из-за посадок судов на мель. Число навигационных аварий можно снизить, повысив точность управления судном при выполнении им поворотов, для чего необходимо учитывать его динамику.

Анализ последних достижений и публикаций, в которых начато решение данной проблемы и выделение нерешенных ранее частей общей проблемы

В работе [1] рассмотрены векториальные погрешности, возникающие при повороте судна. Получены аналитические выражения для погрешностей, которые появляются из-за неточностей при перекладке пера руля и момента начала поворота. Влияние погрешности перекладки пера руля на точность поворота судна с учетом влияния его динамики на вращательное движение рассмотрено в работе [2], а в работе [3] приведен способ формирования области опасных курсов судов с учетом их динамических характеристик поворотливости.

Формулировка целей статьи (постановка задачи) Целью статьи является анализ влияния динамической модели вращательного движения судна прогноза его поворота на безопасность маневрирования судна в стесненных районах.

Изложение основного материала исследования с обоснованием полученных научных результатов

Как показано в работе [1], при повороте судна одной из причин появления векториальной погрешности $S^{(\beta)}$ относительно прогнозируемой точки выхода судна на новый курс является погрешность перекладки пера руля $\Delta \beta_{\mathrm{k}}$.

В случае появления погрешности $\Delta \beta_{\mathrm{k}}$ угол кладки пера руля равен $\beta_{\mathrm{k}}+\Delta \beta_{\mathrm{k}}$, и к концу маневра, когда курса судна изменяется с величины $\mathrm{K}_{\mathrm{o}}$ до значения $\mathrm{K}_{\mathrm{y}}$, погрешность $S^{(\beta)}$ определяется величиной отрезка между прогнозируемой $\mathrm{N}$ и реальной $\mathrm{M}$ точками выхода судна на новый курс, а ее составляющие $S_{x}^{(\beta)}$ и $S_{y}^{(\beta)}$ равны [1]: 


$$
S_{x}^{(\beta)}=x_{N}-x_{M} \text { и } S_{y}^{(\beta)}=y_{N}-y_{M},
$$

где $x_{M}$ и $y_{M}$ - координаты точки М при наличии погрешности $\Delta \beta_{\mathrm{k}}$;

$x_{N}$ и $y_{N}$ - координаты точки $\mathrm{N}$ при отсутствии погрешностей.

Причем погрешность $S^{(\beta)}$ зависит от степени адекватности динамической модели вращательного движения реальному процессу поворота судна.

В работе [2] показано, что сближение двух судов характеризуется областью опасных курсов $\Omega$, которая отображается на плоскости курсов судов. Верхней и нижней границами области $\Omega$ являются точки $\left(K_{1}, K_{2}\right)$, которые соответственно удовлетворяют уравнениям:

$$
\begin{aligned}
& K_{2}^{(1)}=\gamma^{(1)}+\arcsin \left[\frac{V_{1}}{V_{2}} \sin \left(K_{1}-\gamma^{(1)}\right)\right] ; \\
& K_{2}^{(2)}=\gamma^{(2)}+\arcsin \left[\frac{V_{1}}{V_{2}} \sin \left(K_{1}-\gamma^{(2)}\right)\right],
\end{aligned}
$$

где $\gamma^{(1,2)}=\alpha \pm \arcsin D_{d} / D ; \alpha$ и $D$ - соответственно пеленг и дистанция между судами; $D_{d}$ - предельно-допустимая дистанция сближения.

Если точка $\left(K_{1}, K_{2}\right)$ находится на границе или вне области опасных куров $\Omega$ угроза столкновения отсутствует $\left(D_{\min } \geq D_{d}\right)$, в противном случае дистанция кратчайшего сближения $D_{\min }$ меньше предельно-допустимой дистанции $D_{d}$, и сближение судов является опасным. Для безопасного расхождения необходимо выбрать точку $\left(K_{1 y}, K_{2}\right)$, соответствующую курсу уклонения оперирующего судна $K_{1 y}$, которая находится на границе области опасных курсов $\Omega$.

Если рассчитать границы области опасных курсов $\Omega$ с помощью последних выражений, то не будет учтена инерционность судна при повороте, и расхождение произойдет не в предельно-допустимой дистанции $D_{d}$, а на величину $\Delta D_{d}$ ближе [2]. Следовательно, для учета инерционности поворота необходимо предельно-допустимую дистанцию увеличивать до значения $D_{d}+\Delta D_{d}$ и суда разойдутся на дистанции $D_{d}$. Величина поправки $\Delta D_{d}$ однозначно определяется динамической моделью вращательного движения судна и приближение ее значения к истинному определяется степенью адекватности динамической модели вращательного движения реальному процессу поворота судна. Таким образом, в рассмотренных случаях использования модели поворотливости судна определяющим обстоятельством является степень соответствия динамической модели вращательного движения судна реальному процессу его поворота.

В работе [4] рассмотрены различные модели вращательного движения судна, анализ которых показывает, что наиболее адекватная динамическая модель вращательного движения судна описывается следующим дифференциальным уравнением:

$$
\mathrm{T}_{1} \mathrm{~T}_{2} \dddot{\mathrm{K}}+\left(\mathrm{T}_{1}+\mathrm{T}_{2}\right) \ddot{\mathrm{K}}+\dot{\mathrm{K}}=\mathrm{K}_{\omega} \beta_{\mathrm{k}},
$$


где $\mathrm{T}_{1}$ и $\mathrm{T}_{2}$ - постоянные времени, характеризующие инерционные свойства судна;

$\beta_{\mathrm{k}}$ - угол кладки руля;

$\mathrm{K}_{\omega}$ - коэффициент эффективности руля.

Решение приведенного дифференциального уравнения имеет следующий вид:

$$
\mathrm{K}=\mathrm{K}_{\mathrm{o}}+\omega_{\mathrm{r}} \mathrm{t}+\left(\omega_{\mathrm{o}}-\omega_{\mathrm{r}}\right)\left\{\mathrm{T}_{1}^{2}\left[1-\exp \left(-\mathrm{t} / \mathrm{T}_{1}\right)\right]-\mathrm{T}_{2}^{2}\left[1-\exp \left(-\mathrm{t} / \mathrm{T}_{2}\right)\right]\right\} /\left(\mathrm{T}_{1}-\mathrm{T}_{2}\right),
$$

где $\omega_{\mathrm{o}}$ и $\omega_{\mathrm{r}}$ - соответственно начальное значение угловой скорости поворота и частное решение исходного дифференциального уравнения.

Поворот судна содержит две фазы кладки пера руля. Сначала, на первой фазе, в начальный момент времени производится перекладка руля на угол $\beta_{\mathrm{k}}$ и руль удерживается в таком положении в течение интервала времени $\Delta \mathrm{t}_{\mathrm{k}}$. Затем производится перекладка руля на противоположный борт на ту же величину и гасится инерция поворота судна в течение интервала времени $\Delta \mathrm{t}$, по истечению которого судно выходит на заданный курс, угловая скорость поворота обращается в нуль, а перо руля приводится в диаметральную плоскость судна. На первой фазе поворота, длительность которой составляет интервал времени $\Delta \mathrm{t}_{\mathrm{k}}$, выражение (1) приобретает следующий вид:

$$
\mathrm{K}=\mathrm{K}_{\mathrm{o}}+\mathrm{a}_{\omega}\left\{\mathrm{t}-\left\{\mathrm{T}_{1}^{2}\left[1-\exp \left(-\mathrm{t} / \mathrm{T}_{1}\right)\right]-\mathrm{T}_{2}^{2}\left[1-\exp \left(-\mathrm{t} / \mathrm{T}_{2}\right)\right]\right\} /\left(\mathrm{T}_{1}-\mathrm{T}_{2}\right)\right\} .
$$

На второй фазе поворота происходит перекладка руля на противоположный борт на угол $-\beta_{\mathrm{k}}$ и в течение интервала времени $\Delta \mathrm{t}$ происходит одерживание судна. Для этой фазы поворота значение текущего курса описывается зависимостью:

$$
\begin{aligned}
\widetilde{\mathrm{K}}=\mathrm{K} & -\mathrm{a}_{\omega} \mathrm{t}+\mathrm{a}_{\omega}\left\{2-\left[\mathrm{T}_{1} \exp \left(-\Delta \mathrm{t}_{\mathrm{k}} / \mathrm{T}_{1}\right)-\mathrm{T}_{2} \exp \left(-\Delta \mathrm{t}_{\mathrm{k}} / \mathrm{T}_{2}\right)\right] /\left(\mathrm{T}_{1}-\mathrm{T}_{2}\right)\right\} \times \\
& \times\left\{\mathrm{T}_{1}^{2}\left[1-\exp \left(-\mathrm{t} / \mathrm{T}_{1}\right)\right]-\mathrm{T}_{2}^{2}\left[1-\exp \left(-\mathrm{t} / \mathrm{T}_{2}\right)\right]\right\} /\left(\mathrm{T}_{1}-\mathrm{T}_{2}\right) .
\end{aligned}
$$

Для вычисления интервалов времени $\Delta \mathrm{t}_{\mathrm{k}}$ и $\Delta \mathrm{t}$ необходимо составить систему уравнений, которая в общем случае формализует требования поворота на заданное приращение курса $\Delta \mathrm{K}$, а также обращение в нуль угловой скорости на момент времени выхода на новый курс и имеет следующий вид:

$$
\left\{\begin{array}{l}
\Delta \mathrm{K}=\mathrm{K}\left(\Delta \mathrm{t}_{\mathrm{k}}\right)+\widetilde{\mathrm{K}}(\Delta \mathrm{t}) \\
\omega\left(\Delta \mathrm{t}_{\mathrm{k}}, \Delta \mathrm{t}\right)=0
\end{array} .\right.
$$

С учетом выражений (2) и (3) и системы уравнений (4) находим выражения для расчета интервалов времени $\Delta \mathrm{t}_{\mathrm{k}}$ и $\Delta \mathrm{t}$ методом простых итераций с начальным приближением $\Delta \mathrm{t}_{\mathrm{k}}=\Delta \mathrm{K} / \mathrm{a}_{\omega}$ : 


$$
\begin{gathered}
\Delta \mathrm{t}_{\mathrm{k}}=\Delta \mathrm{t}+\left\{\mathrm{T}_{1}^{2}\left[1-\exp \left(-\Delta \mathrm{t}_{\mathrm{k}} / \mathrm{T}_{1}\right)\right]-\mathrm{T}_{2}^{2}\left[1-\exp \left(-\Delta \mathrm{t}_{\mathrm{k}} / \mathrm{T}_{2}\right)\right]\right\} /\left(\mathrm{T}_{1}-\mathrm{T}_{2}\right)- \\
-\left\{2-\left[\mathrm{T}_{1} \exp \left(-\Delta \mathrm{t}_{\mathrm{k}} / \mathrm{T}_{1}\right)-\mathrm{T}_{2} \exp \left(-\Delta \mathrm{t}_{\mathrm{k}} / \mathrm{T}_{2}\right)\right] /\left(\mathrm{T}_{1}-\mathrm{T}_{2}\right)\right\} \times \\
\left\{\mathrm{T}_{1}^{2}\left[1-\exp \left(-\Delta \mathrm{t} / \mathrm{T}_{1}\right)\right]-\mathrm{T}_{2}^{2}\left[1-\exp \left(-\Delta \mathrm{t} / \mathrm{T}_{2}\right)\right]\right\} /\left(\mathrm{T}_{1}-\mathrm{T}_{2}\right)+\Delta \mathrm{K} / \mathrm{a}_{\omega} . \\
\Delta \mathrm{t}=-\mathrm{T}_{1} \ln \left\{\left(\mathrm{T}_{2} / \mathrm{T}_{1}\right) \exp \left(-\Delta \mathrm{t} / \mathrm{T}_{2}\right)+\left[\left(\mathrm{T}_{1}-\mathrm{T}_{2}\right) / \mathrm{T}_{1}\right] \times\right. \\
\left.\times\left\{2-\left[\mathrm{T}_{1} \exp \left(-\Delta \mathrm{t}_{\mathrm{k}} / \mathrm{T}_{1}\right)-\mathrm{T}_{2} \exp \left(-\Delta \mathrm{t}_{\mathrm{k}} / \mathrm{T}_{2}\right)\right] /\left(\mathrm{T}_{1}-\mathrm{T}_{2}\right)\right\}^{-1}\right\} .
\end{gathered}
$$

Таким образом, для расчета величин $\Delta \mathrm{t}_{\mathrm{k}}$ и $\Delta \mathrm{t}$ методом простых итераций, задаваясь предыдущим значением $\Delta \mathrm{t}_{\mathrm{k}}$, с помощью выражения (6) вычисляется значение $\Delta \mathrm{t}$, которое затем подставляется в выражение (5) для расчета последующего значения $\Delta \mathrm{t}_{\mathrm{k}}$.

Для определения погрешности $S^{(\beta)}$ необходимо определить координаты точек M и N. Координаты точки М определяются выражениями:

$$
\begin{aligned}
& x_{M}=\int_{0}^{\Delta \mathrm{tk}} \mathrm{V}_{\mathrm{o}} \sin \left[\mathrm{K}_{\mathrm{o}}+\mathrm{K}\right] \mathrm{dt}+\int_{0}^{\Delta \mathrm{t}} \mathrm{V}_{\mathrm{o}} \sin \left[\mathrm{K}_{\mathrm{o}}+\mathrm{K}\left(\Delta \mathrm{t}_{\mathrm{k}}\right)+\tilde{\mathrm{K}}\right] \mathrm{d} \mathrm{t}, \\
& y_{M}=\int_{0}^{\Delta \mathrm{tk}} \mathrm{V}_{\mathrm{o}} \cos \left[\mathrm{K}_{\mathrm{o}}+\mathrm{K}\right] \mathrm{d} \mathrm{t}+\int_{0}^{\Delta \mathrm{t}} \mathrm{V}_{\mathrm{o}} \cos \left[\mathrm{K}_{\mathrm{o}}+\mathrm{K}\left(\Delta \mathrm{t}_{\mathrm{k}}\right)+\tilde{\mathrm{K}}\right] \mathrm{dt},
\end{aligned}
$$

причем в выражениях (2), (3), (5) и (6) применяем $\mathrm{a}_{\omega}=\mathrm{k}_{\omega} \beta_{\mathrm{k}}$.

Аналогично находятся выражения для координат $x_{N}$ и $y_{N}$ точки $\mathrm{N}$, только при их расчете по формулам (7) и (8) значение $\mathrm{a}_{\omega}=\mathrm{k}_{\omega}\left(\beta_{\mathrm{k}}+\Delta \beta_{\mathrm{k}}\right)$. При расчете координат с помощью (7) и (8) определенные интегралы, которые входят в эти формулы, не выражаются в элементарных функциях и их значения находятся численными методами, например, используется метод Симпсона.

Аналогично, расчет величины поправки $\Delta D_{d}$ также производится с использованием расчета координат судна с помощью выражений (7) и (8), что обеспечивает безопасное расхождение опасно сближающихся судов методом их внешнего управления.

\section{Выводы и перспектива дальнейшей работы по данному направлению}

Таким образом, минимизация рисков при плавании судна в стесненных условиях достигается при использовании рассмотренной в работе динамической модели вращательного движения судна третьего порядка.

В дальнейшем следует рассмотреть целесообразность использования моделей вращательного движения судна с учетом времени перекладки пера руля. 


\section{ЛИТЕРАТУРА}

1. Ворохобин И.И. Векториальные погрешности, возникающие при повороте судна/ Ворохобин И.И., Казак Ю.В. // Судовождение: Сб. научн. трудов. / OНМА, Вып. 26. - Одесса: «ИздатИнформ», 2015 - С.

2. Калиниченко Г.Е. Формирование области опасных курсов судов с учетом их динамических характеристик / Калиниченко Г.Е., Пасечнюк С.С. // Автоматизация судовых технических средств. - 2017. - № 23 - С. 28 - 33 .

3. Казак Ю.В. Влияние погрешности перекладки пера руля на точность поворота судна // Судовождение: Сб. научн. трудов. / ОНМА, Вып. 27. Одесса: «ИздатИнформ», 2017 - С. 96-100.

4. Вагущенко Л.Л. Судно как объект автоматического управления/ Л.Л. Вагущенко - Одесса: ОГМА, 2000. - 140 с. 